\title{
An Electromagnetic Resonance Based Interpretation of Quantum Theory
}

\author{
Thomas A. Kriz \\ Alpha Omega Advanced Studies, Cedar Park, USA \\ Email address: \\ t_kriz@yahoo.com
}

To cite this article:

Thomas A. Kriz. An Electromagnetic Resonance Based Interpretation of Quantum Theory. American Journal of Modern Physics. Vol. 4, No. 3, 2015, pp. 125-131. doi: 10.11648/j.ajmp.20150403.14

\begin{abstract}
An electromagnetic (EM) resonance based model derived from Maxwell's Equations is used with constraint conditions to characterize the quantum properties of both matter particles and photons. The model, as constrained by integer spin-orbit ratio, integer multiples of Planck's constant, angular momentum balance, charge balance, and EM resonance form, yields analytical results that are comparable to those from traditional quantum mechanics (QM), and electrodynamics (QED), but obtained with reduced analytical effort. EM compound resonance models are used to characterize quantum chromodymanics (QCD) quarks in neutrons and protons. It is also shown that EM resonance models give evidence that supports QCD “color-confinement” and "color-change” concepts. Analysis is limited to steady-state resonance forms.
\end{abstract}

Keywords: Maxwell's Equations, Electromagnetic Resonance, Quantum Mechanics, Quantum Electrodynamics, Quantum Chromodynamics

\section{Introduction}

The "Modern Physics" era can be characterized as one dominated by the development of analytical "behavioral models". In this regard, Feynman spoke well when he said: "It is not philosophy we are after, but the behavior of real things". ${ }^{1}$ Indeed, during the twentieth century, the practice of developing causally based physical models was largely abandoned in favor developing mathematical descriptions that described observed physical behavior. The risk one takes when developing such a "behavioral" model, however, is that "one may be trying to solve a puzzle with missing pieces". The observable data set used in the behavioral model may lack "hidden variables" information that is necessary to analytically portray system state and change of state accurately. ${ }^{2,3}$ Complexity of analytical solution procedures is another potential problem. An analytical model may depend upon limitations imposed by the "available information set" in a way that can impact the complexity of its solution procedures. In this regard, the paper presented here describes a causally based EM resonance solution approach that aims at reducing the complexity of quantum theory solution procedures. Specifically, an EM resonance model derived from Maxwell's Equations is used with constraint conditions to obtain quantum solutions that otherwise would be available only from traditional quantum methods. As an Initial step, historical quantum theory developments are briefly reviewed to identify where an opportunity to use such EM based information was overlooked.

\section{A Brief Summary of Early Quantum Theory Developments}

The birth of the quantum age began in the year 1900, when Max Planck discovered that radiated electromagnetic energy $E$ could only be emitted in full-wave form ${ }^{4,5}$

$$
\mathrm{E}=h v=h c \lambda_{\mathrm{r}}
$$

where $h$ is Planck's constant, $v$ is radiation frequency, $\mathrm{c}$ is the speed of light in free space, and $\lambda_{\mathrm{r}}$ is the wavelength of the radiation. Later practice simply referred to such radiated energy quanta as a photons. In 1877, Boltzmann at the University of Vienna had published a theoretical paper where he conjectured that the energy states of a physical system could be discrete. Planck considered this possibility in developing the result shown in Eq. (1), and defined the basic set of physical units that can still be found in most quantum theory models.

However, during the $1920 \mathrm{~s}$, both Planck and Einstein, perceiving that newly developed models in quantum 
mechanics were incompletely defined, pursued alternative approaches to a unified field theory.

The breakthrough event that enabled further development of quantum theory, however, came in 1924 from de Broglie, who wrote a one page $\mathrm{PhD}$ thesis at the Sorbonne in Paris. The deBroglie thesis, reviewed and approved by Einstein, asserted that a matter particle energy $\mathrm{mc}^{2}$ was equal to Planck photon energy ${ }^{6,7}$

$$
\mathrm{mc}^{2}=h \mathrm{v}=h c / \lambda_{\mathrm{r}},
$$

and had a matter field wavelength defined by

$$
\lambda_{\mathrm{m}}=h / \mathrm{p}=(h / \mathrm{mv})\left(1-\mathrm{v}^{2} \mathrm{c}^{2}\right)^{1 / 2}
$$

where $m$ is particle rest mass, $\mathrm{v}$ is matter wave velocity, and $p$ is particle momentum. This provided enough previously "hidden variables" information to show a minimal connection between photons and matter particles. Nevertheless, it was sufficient for Schrödinger to develop a new matter wave equation, while recovering from pneumonia at a sanatorium in Arosa, Austria in $1925 .{ }^{8}$

Unfortunately, an opportunity to show an even stronger connection between photons and matter was missed. Had the quantum researchers been familiar with microwave solutions to Maxwell's Equations, they might have developed resonance cavity solution forms that were not found until twenty years later. Such solutions were developed at the MIT Radiation Laboratory, ${ }^{9}$ and by similar laboratories in Germany and Japan, for use in RADAR systems during World War II. However, since such research was classified SECRET, it was not publicly known until well after the war. As will be shown in this presentation, however, knowledge of such EM resonance forms can be of value in reducing the complexity of quantum solution procedures.

\section{A Particle Focused EM Resonance Template}

A previously published paper by our group, ${ }^{10}$ gives strong evidence that there is a close relationship between quantum waves and EM waves, and furthermore suggests a close relationship between photons and matter. Such a view is also supported in a template, ${ }^{11}$ that can map theoretical EM resonance properties to matter orbital and spin attributes. This template is developed as follows. Given a harmonic EM waveform propagating in a loss-less medium with frequency $\omega$ and electric field intensity $\mathrm{E}=\mathrm{E}_{0} \mathrm{e}^{\mathrm{i} \omega \mathrm{t}}$ and magnetic field intensity $\mathrm{H}=\mathrm{H}_{0} \mathrm{e}^{\mathrm{i} \omega \mathrm{t}}$ where $\mathrm{E}_{0}$ and $\mathrm{H}_{0}$ are field amplitude constants, and $\mathrm{i}=(-1)^{1 / 2}$, the appropriate governing form of Maxwell's Equations is defined by the EM wave equation pair that assumes no existing matter

$$
\begin{gathered}
\nabla^{2} \mathrm{E}+\omega^{2} \epsilon_{0} \mu_{0} \mathrm{E}=0 \text {, and } \\
\nabla^{2} \mathrm{H}+\omega^{2} \epsilon_{0} \mu_{0} \mathrm{H}=0
\end{gathered}
$$

where $\epsilon_{0}$ is the dielectric constant in free space, and $\mu_{0}$ is the permeability in free space. Within this framework, the resonance form must support the appropriate number of EM spins to be synchronous with traveling wave resonance at velocity

$$
\mathrm{v}=\mathrm{c} / \beta,
$$

where $\beta$ is a factor which defines the number of EM full waves (eg; the number of spins), and $c$ is the speed of light looking backward and forward along the traveling wave to yield respectively the observed backward and forward frequencies ${ }^{11}$

$$
\begin{gathered}
\omega_{\mathrm{b}}=\omega[(\mathrm{c}-\mathrm{v}) /(\mathrm{c}+\mathrm{v})]^{1 / 2}, \text { and } \\
\omega_{\mathrm{f}}=\omega[(\mathrm{c}+\mathrm{v}) /(\mathrm{c}-\mathrm{v})]^{1 / 2} .
\end{gathered}
$$

Now, let

$$
\begin{gathered}
\zeta \omega_{\mathrm{b}}=(\beta-1) \omega,(\text { backward resonance }), \text { and } \\
\zeta \omega_{\mathrm{f}}=(\beta+1) \omega,(\text { forward resonance }) .
\end{gathered}
$$

Then, eliminating $\omega$, one obtains

$$
\zeta^{2}=\beta^{2}\left[1-(\mathrm{v} / \mathrm{c})^{2}\right]=\beta^{2}-1
$$

which defines the relativistic relationship between $\zeta$ and $\beta$ where the constraints on $\beta, \beta \pm 1$, and $v$ are determined by the specific form of the resonance. Eq. (11) can then be interpreted in terms of a variety of particular resonance forms shown in the following: ${ }^{11}$

- $\beta=0$, a meson spin-0 particle with $v=v p=c 2 / v g \rightarrow \infty$ as $v g \rightarrow 0$, and $\zeta^{2}=-1$,

- $\beta=1 / 2$, a spin- $1 / 2$ particle with $v=v g=c / 2, v p=c 2 / v g=2 c$, and $\zeta^{2}=-3 / 4$,

- $\beta=1$, a spin-1 photon particle with velocity $v=c$ and $\zeta^{2}=0$,

- $\beta=N, \quad(N \geq 2 \quad \& \quad N \pm 1$ integers), an orbiting electron particle with $v=c / N<c$, and $\zeta^{2}=N 2-1$, and

- $\beta \rightarrow \infty$, a motionless free electron particle with orbital velocity $v \rightarrow 0$ and $\zeta^{2} \rightarrow \infty$,

where $\beta$ is a spin attribute, and $v p$ and $v g$ are the phase wave and group wave velocity respectively of a wave packet focused set of harmonic components. Microwave analogs may then be defined for each of the above defined $\beta$ categories. ${ }^{10,11}$ Analytically, these categories must be constrained by integer spin-orbit ratio, integer multiples of Planck constant, angular momentum balance in ground state, charge balance, and EM resonance form, to obtain quantitative results that are limited to steady state resonance forms.

\section{Some Analytical Results}

\subsection{Quantum Mechanics}

In spherical polar coordinates, $\Psi=R \Theta \Phi$, the steady-state form of the Schrödinger equation for Hydrogen atom is the differential equation ${ }^{12}$

$$
\begin{gathered}
\left(\sin ^{2} \theta / \mathrm{R}\right)\left[d / d \mathrm{r}\left(\mathrm{r}^{2} \mathrm{dR} / d \mathrm{r}\right)\right]+(\sin \theta / \Theta)[d / d \theta(\sin \theta \mathrm{d} \Theta / d \theta)]+\Phi \\
{ }^{1} \mathrm{~d}^{2} \Phi / \mathrm{d} \phi^{2}+\left(2 \mathrm{mr}^{2} \sin ^{2} \theta / h^{2}\right)\left[\left(\mathrm{e}^{2} / 4 \pi \epsilon_{0}\right)+\mathrm{E}\right]=0
\end{gathered}
$$


where $R(r)$ describes how $\Psi$ varies radially with $\theta$ and $\phi$ constant, $\Theta(\theta)$ describes how $\Psi$ varies with zenith angle $\theta$ along a sphere centered meridian with $r$ and $\phi$ constant, and $\Phi$ describes how $\Psi$ varies with azimuth angle $\phi$ along a sphere centered parallel with $r$ and $\theta$ constant. Solving Eq. (12), one obtains the three quantum numbers: ${ }^{13}$

Principal quantum number $n=1,2,3 \ldots$ where $\mathrm{E}_{\mathrm{n}}=\mathrm{E}_{1} / \mathrm{n}^{2}$ (orbital kinetic energy)

Orbital quantum number $l=0,1,2,3 \ldots(n-1)$ where $\mathrm{L}=[l(l-1)]^{1 / 2} h$ (angular momentum)

Magnetic quantum number $m_{r}=0, \pm 1, \pm 2 \ldots . \pm l$ where $\mathrm{L}_{\mathrm{Z}}=m_{r} h$ (magnetic field vector)

As shown, the kinetic energy level of an orbiting electron is inversely proportional to the square of the Principle quantum number. At ground level (eg; $n=1)$, the electron kinetic energy $\mathrm{E}_{1}=-13.60 \mathrm{eV}$ and diminishes to almost zero as the electron orbit reaches the mass shell of the atom. Magnetic quantum number, on the other hand, essentially obeys Maxwell's Equation for an orbiting charged particle with angular momentum that can be parallel or anti-parallel to an applied magnetic field. It therefore is equal to orbital quantum number

$$
\mathrm{m}_{\mathrm{r}}= \pm l= \pm(\mathrm{n}-1) .
$$

Orbital quantum number, on the other hand, is more complex. At ground state level, $l=0$, therefore implying that orbiting electron angular momentum appears to be fully balanced by the opposite angular moment of the proton nucleus. Above ground state, however, it appears that the angular moment of the particle is not completely balanced until electron orbit reaches the hydrogen particle mass shell. At such a point, the orbiting electron is essentially free. It is worth noting, however, that angular momentum of the particle is ${ }^{13}$

$$
\mathrm{L}=[l(1-1)]^{1 / 2} h .
$$

Since Eq. (17) defines a fractional form of Planck's constant, the result is open to question as a steady state form.

By comparison, constraints for the above defined EM resonance template require an electron with an integer number of spins per orbit and an integer multiple of Planck's constant. In this regard, consider the orbiting electron of a Hydrogen atom in its ground energy state with a fine structure (spin-orbit coupling) constant $\sigma^{-1}$. A commonly quoted fine structure (eg; spin/orbit) value is approximately $137.03604,{ }^{14}$ which unfortunately views the locus of the atomic nucleus as fixed in space. Clearly, such a quoted value is not an integer. It should be noted, however, that using the electron "reduced mass" method, the quoted fine structure value may be corrected to satisfy the integer number of spins per orbit cited above. Making such a correction ${ }^{11}$

$$
\alpha^{-1}=137.03604\left(\mathrm{~m}_{\mathrm{e}}{ }^{\prime} / \mathrm{m}_{\mathrm{e}}\right)^{1 / 2} \approx 137.0000
$$

where $m_{e}$ is the mass of an orbiting electron, $m_{p}$ is the mass of the nucleus, $\mathrm{m}_{\mathrm{e}}$ ' is the reduced electron mass, $m_{e}{ }^{\prime} / m_{e}=m_{p} /\left(m_{p}+m_{e}\right)=0.99945$. ${ }^{15}$ Viewing the electron orbit locus as a "slow wave" structure with Bohr radius $r_{e}=\lambda_{c} \alpha^{-}$ ${ }^{1} / 2 \pi$ where $\lambda_{c}$ is the electron Compton wave length, one obtains a resonance condition with approximately 137 electron spins per orbit at base orbit. In this case, $\beta=\alpha^{-1}=137$ (the corrected spin-orbit coupling constant), with $(\beta+1)=$ 138 looking forward, and $(\beta-1)=136$ looking backwards. But, what about the orbits with numbers below 137? Note here also that electron orbital velocity at the base level is $\mathrm{c} / 137$, and that its angular momentum value must be the lowest integer multiple of Planck constant, simply $h$. Since the orbits below that the level $\beta<\alpha^{-1}=137$ would therefore have an angular momentum values that are a fraction of $h$, they are invalid. Orbit levels with $\beta>\alpha^{-1}=137$ also have gaps as result of the integer multiple of $h$ requirement and due to particle kinetic and potential energy mismatch. In this case, all valid orbit levels would be those that are an integer multiples of a 137 spin orbit value and correspond to $n h$ where $n$ is an integer.

Now consider electron kinetic energy $E_{l}$ at ground level that can be represented by

$$
\mathrm{E}_{1}=\mathrm{m}_{\mathrm{e}} \mathrm{v}^{2} / 2=\mathrm{m}_{\mathrm{e}}\left(\mathrm{c} / \alpha^{-1}\right)^{2} / 2 .
$$

where $\mathrm{v}$ is electron orbital velocity $c / \alpha^{-1}$. At the $n t h$ level, this becomes

$$
\mathrm{E}_{\mathrm{n}}=\mathrm{m}_{\mathrm{e}}(\mathrm{nv})^{2} / 2=\mathrm{m}_{\mathrm{e}}\left(\mathrm{c} / \mathrm{n} \alpha^{-1}\right)^{2} / 2,
$$

Thus, it can be seen then that $E_{n}=E_{1} / n^{2}$, the same as traditional QM. Eq.(17) therefore uniquely defines the only other electron orbital states that can be validly occupied (eg; $\beta=n^{2} \alpha^{-1}$ ).

Other quantum numbers also depend directly on $n$. If there were no impact from the proton nucleus, the orbital quantum number $l$ could be expected to be integer multiples of Planck's constant $h$, and therefore equal to $n$. However, since angular momentum must balance when $n=1$, the proton impact must generally be equal and opposite to that of the orbiting electron when $l=0$ (eg; be equal to $-h$ ). Since the proton nucleus must also have the minimum existing integer number of spins, its spin-orbit ratio is uniquely equal to one. Therefore, the proton has no significant orbital attribute as shown below in Eq. (18), and its orbital quantum impact is independent is of the electron orbital state. Therefore, $l=n-1$. Angular momentum balance of the Hydrogen atom, however, fails as a stable resonance form above the ground state, due to the $l=n-1$ attribute.

From Maxwell's Equations, an orbiting charged particle constitutes a source of magnetism. Thus, it can be expected to generate a magnetic field vector $L_{Z}$ in a direction $\mathrm{z}$ that can be associated with the orbital quantum number $l$. The vector direction $\mathrm{z}$ is defined by the presence of an external magnetic field can be parallel or anti-parallel to the applied external field. Thus, the implied Magnetic quantum value for the EM resonance chart must be equivalent to $m_{r}= \pm 1= \pm(n-1)$ with multiple integer values of $h$.

From the foregoing then, it can be seen that the results derived via the constraints on the EM resonance template 
given above substantially agree with the Schrödinger equation results. The orbiting electron resonance case just described can be termed a "Simple Resonance" method since it can be defined by single orbital resonance tones. It can happen, however, that a "Compound Resonance" set may be required to define a valid orbital resonance condition, that otherwise may appear invalid as a Simple resonance. This is the exactly case for the proton nucleus of the Hydrogen atom at the ground state. As will be shown, the form must be defined as a quark based Compound Resonance. ${ }^{11}$

\subsection{Quantum Chromodynamics}

To begin, consider the equation for balanced angular momentum at ground state in a Hydrogen atom:

$$
\mathrm{pr}=\mathrm{mvr}=\mathrm{m}_{\mathrm{e}}\left(\mathrm{c} / \alpha^{-1}\right) \mathrm{r}_{\mathrm{e}}=\mathrm{m}_{\mathrm{p}}\left(\mathrm{c} / \tilde{\alpha}^{-1}\right) \mathrm{r}_{\mathrm{p}}=\hbar,
$$

where $r_{e}$ is the Bohr radius of the electron orbit, $r_{p}$ is the radius of proton orbit, $\tilde{\alpha}^{-1}$ represents the spin/orbit coupling constant for proton orbital resonance and $\hbar=h / 2 \pi$ the reduced Planck constant. Note, however, that charge based electrostatic attraction can significantly impact angular momentum balance in the atom. A corrected form of Eq. (18) must therefore be used to determine information about an "equivalent" orbital resonance condition of the proton nucleus in the atom. Moreover, a Compound Resonance form is also required to validly define the orbital resonance conditions of the neutron based quark components.

Recall that the Bore radius $a_{0}=5.3 \times 10^{11} \mathrm{~m}$ in the ground state requires a balance between kinetic energy $m_{e} v^{2} / 2$ and potential energy due to electrostatic attraction $\left(e^{2} / 4 \pi \epsilon_{0} r^{2}\right)$. Also note that charge balance in the Hydrogen atom can be defined by:

$$
\mathrm{e}^{2}=\hbar \mathrm{c} / \alpha^{-1}=\mathrm{m} \lambda_{\mathrm{c}} \mathrm{c}^{2} / \alpha^{-1}
$$

where $\lambda_{C}=\hbar / m c$ is the Compton wavelength, and $m$ is the rest mass of a charged particle. Since electron charge $e_{e}$ and proton charge $e_{p}$ must balance, Eq. (19) implies that

$$
\mathrm{e}_{\mathrm{e}}{ }^{2}=\mathrm{m}_{\mathrm{e}} \lambda_{\mathrm{ce}} \mathrm{c}^{2} / \alpha^{-1}=\mathrm{e}_{\mathrm{p}}{ }^{2}=\mathrm{m}_{\mathrm{p}} \lambda_{\mathrm{cp}} \mathrm{c}^{2} / \tilde{\alpha}^{-1}
$$

where $\lambda_{c p}$ is the Compton wavelength of the proton, and an equivalent electron wavelength defined by:

$$
\lambda_{\text {ce }} / 2 \pi=\mathrm{a}_{0} .
$$

Since the proton has no significant orbital motion, proton spin orbit ratio $\tilde{\alpha}^{-1}=1$, and one may define an "equivalent" proton orbit wavelength, that takes into account the impact of particle electrostatic attraction, as

$$
\lambda^{\prime}{ }_{\mathrm{cp}} / 2 \pi \approx \mathrm{a}_{0} / 1836 \alpha^{-1} \approx \mathrm{r}_{\mathrm{p}}{ }^{\prime} .
$$

Assume then that QCD values for mass and charge are correct with quark "up" and "down" for charge and mass defined as ${ }^{16.17}$

$$
\begin{aligned}
& \mathrm{m}_{\mathrm{u}}=\mathrm{m}_{\mathrm{d}}=\mathrm{m}_{\mathrm{p}} / 3 \\
& \mathrm{e}_{\mathrm{p}}{ }^{2}=2 \mathrm{e}_{\mathrm{u}}{ }^{2}+\mathrm{e}_{\mathrm{d}}{ }^{2}
\end{aligned}
$$

$$
\mathrm{e}_{\mathrm{u}}{ }^{2}=\left(2 / 3 \mathrm{e}_{\mathrm{p}}\right)^{2}, \mathrm{e}_{\mathrm{d}}{ }^{2}=\left(1 / 3 \mathrm{e}_{\mathrm{p}}\right)^{2},
$$

where $\mathrm{e}_{\mathrm{u}}^{2}$ defines the "up" quark charge, $\mathrm{e}_{\mathrm{d}}^{2}$ defines "down" quark charge, and $\mathrm{m}_{\mathrm{u}}$, mass for quark "up", and $\mathrm{m}_{\mathrm{d}}$, quark "down". The quark attributes for the proton Compound quark resonance components must fulfill the requirements for a valid orbital resonance defined in the following. ${ }^{11}$

$$
\begin{array}{r}
\mathrm{e}_{\mathrm{u}}^{2}=4 \mathrm{~m}_{\mathrm{p}} \lambda_{\mathrm{cp}}{ }^{2} \mathrm{c}^{2} / 9 \alpha^{-1}=\left(2 \mathrm{e}_{\mathrm{p}} / 3\right)^{2}, \\
\lambda_{\mathrm{u}} / 2 \pi \approx 4 \mathrm{r}_{\mathrm{p}}{ }^{\prime} / 9=\left[(4 / 9) \mathrm{r}_{\mathrm{p}}{ }^{\prime} / 1\right], \\
\mathrm{e}_{\mathrm{d}}{ }^{2}=\mathrm{m}_{\mathrm{p}} \lambda_{\mathrm{cp}} \mathrm{c}^{2} / 9 \alpha^{-1}=\left(\mathrm{e}_{\mathrm{p}} / 3\right)^{2}, \text { and } \\
\lambda_{\mathrm{d}} / 2 \pi \approx \mathrm{r}_{\mathrm{p}}{ }^{\prime} / 9=\left[(1 / 3) \mathrm{r}_{\mathrm{p}}{ }^{\prime} / 3\right] .
\end{array}
$$

Eqs. (26-29) show that the orbital locus of a quark "up" is $r_{u}=(4 / 9) r_{p}{ }^{\prime}$ and a quark "down" is $r_{d}=(1 / 3) r_{p}{ }^{\prime}$. This fulfills an expectation that a quark orbital locus must be inside the radius of the proton orbit. It can also be seen from Eqs. (2629 ), that a proton composed of two "up" quarks and a single "down" quark has charge which balances that of an electron

$$
\begin{gathered}
\mathrm{e}_{\mathrm{p}}{ }^{2}=\left[\left(\mathrm{mp} \lambda_{\mathrm{cp}}{ }^{\prime} \mathrm{c}^{2} / 9 \alpha^{-1}\right)^{1 / 2}-2\left(4 \mathrm{mp} \lambda_{\mathrm{cp}}{ }^{\prime} \mathrm{c}^{2} / 9 \alpha^{-1}\right)^{1 / 2}\right]^{2}= \\
{\left[\mathrm{e}_{\mathrm{d}}-2 \mathrm{e}_{\mathrm{u}}\right]^{2}=\mathrm{e}_{\mathrm{e}}{ }^{2},}
\end{gathered}
$$

and a neutron composed of two "down" quarks and a single "up" quark is charge neutral

$$
\begin{gathered}
\mathrm{e}_{\mathrm{n}}{ }^{2}=\left[2\left(\mathrm{mp} \lambda_{\mathrm{cp}}{ }^{\prime} \mathrm{c}^{2} / 9 \alpha^{-1}\right)^{1 / 2}-\left(4 \mathrm{mp} \lambda_{\mathrm{cp}}{ }^{\prime} \mathrm{c}^{2} / 9 \alpha^{-1}\right)^{1 / 2}\right]^{2}= \\
{\left[2 \mathrm{e}_{\mathrm{d}}-\mathrm{e}_{\mathrm{u}}\right]^{2}=0}
\end{gathered}
$$

Eqs. (26-29) also indicate that the quark "up" and "down" constituents of the proton are a valid orbital Compound Resonance per the template requirement defined above, since the positively charged "up" quark has $\beta=1$, and the negatively charged "down" quark, $\beta=3$. When these results are joined to another from a paper by our group ${ }^{18}$ which shows that a halfspin particle can be modeled as a micro-black-hole, this is provides evidence to support the concept of chromodynamic "color confinement". Specifically, one cannot access a quark as an isolated particle because of the micro-black-hole attribute that applies to protons and neutrons. Since discussion here is limited to stable particles, it should be noted that second and third generation quarks such as "charm", "strange", "top" and "bottom" quickly decay to be come "up" and "down" quarks, and therefore are not discussed.

Now consider the concept of chromodynamic "color change". This is frequently defined in terms of the Beta decay process in an isolated neutron population that transforms neutrons into protons. The "standard model" for particles represents such a process as one that converts a neutron "down" quark constituent to an "up" quark, after colliding with a virtual $\mathrm{W}^{-}$boson with large mass, to yield a proton, an electron, and an anti-neutrino. The process is usually portrayed in terms of a QED style "Feynman Diagram" that otherwise would be limited to characterizing only "weak interactions". 19,20 The standard model, however, proceeds to portray such a particle collision result 
behaviorally as a "non-linear" process. More will be said on this point in the next section that deals with QED. The immediate point of focus here, however, is to show what such "color change" means in terms of EM resonance based phenomena.

A convenient microwave analog to characterize the EM resonance form of a half-spin particle is a closed loop torus shaped loss-less cylindrical cavity. ${ }^{11}$ As the shape of the torus loop is tightly closed to make its circumference equal to the wavelength of a trapped photon, it can be perceived as an idealized model for a half-spin particle. From the EM resonance template give above, and a recent paper published by our group that shows equivalence of a half-spin particle to a micro-black hole, ${ }^{18}$ it can be expected that the idealized torus then will tend to take a sphere-like shape. Since spherical mode solutions to Maxwell's Equations in flat space-time are severely restricted, and the following analysis is approached as a limiting solution. The EM field conditions in the torus shaped cylindrical cavity will therefore be approached, by forcing a straight-line section to take the shape of a closed loop torus. Consider a torus shaped cylindrical wave guide cavity with coordinates $(r, \phi, z)$ where the $\mathrm{z}$ axis is located on the circumference of the torus, $r$ is the radial axis of the waveguide cross-section, $\phi$ is an angular offset from a defined r-axis reference, $a$ is the radius of the waveguide cross-section, and the length of the torus shaped cavity along the z-axis is equal to the length of a trapped EM full wave (eg; a photon). The form of Maxwell's Equations that govern such a trapped $E M$ wave in $\mathrm{TE}_{\mathrm{mnk}}$ mode propagation field is ${ }^{21}$

$$
\partial^{2} \mathrm{H}_{\mathrm{z}} / \partial \mathrm{r}^{2}+\mathrm{r}^{-1} \partial \mathrm{H}_{\mathrm{z}} / \partial \mathrm{r}+\mathrm{r}^{-2} \partial^{2} \mathrm{H}_{\mathrm{z}} / \partial \phi^{2}+\mathrm{k}_{\mathrm{c}}^{2} \mathrm{H}_{\mathrm{z}}=0
$$

where $H_{z}$ has the form of a wave propagating in the $z$ direction defined by the expression $H(r, \phi) \exp (-\gamma z+i \omega t)$ with $\gamma$ as the propagation constant, $i=(-1)$ as defined by eigenvalues

$$
\mathrm{k}_{\mathrm{c}}^{2}=\gamma^{2}+\omega^{2} \epsilon \mu \text {. }
$$

It is also assumed that the waveguide walls are loss-less

$$
\left|\partial \mathrm{H}_{\mathrm{z}} / \partial \mathrm{r}\right|_{\mathrm{r}=\mathrm{a}}=0
$$

One possible solution to Eq. (32) then is

$$
\mathrm{H}_{\mathrm{z}}=\mathrm{H}_{1} \mathrm{~J}_{\mathrm{m}}\left(\mathrm{k}_{\mathrm{c}} \mathrm{r}\right) \cos \mathrm{m} \phi
$$

where $\mathrm{J}_{\mathrm{m}}\left(\mathrm{k}_{\mathrm{c}} \mathrm{r}\right)$ is a Bessel function of the first kind of order $m$ with argument $\mathrm{k}_{\mathrm{c}}$, and $\mathrm{H}_{1}$ is a constant for propagated energy magnitude. The solution in Eq. (35) then leads to a $\mathrm{TE}_{01}$ field distribution form that can then be mapped to a neutron as an analog. In such a case, the $\mathrm{TE}_{011}$ mode (see ref 21-Fig. 4-5) cross-section distribution of the electric field is circular and contained within the particle whereas the magnetic field distribution spreads radially and externally from the center. A quark form model is also available as $\mathrm{TE}_{013}$ mode with three similar tiers. As a result of the circular contained electric field distribution, a static neutron analog has no observable charge, but only a rather large magnet moment (eg; -1.93$)^{22}$.

Alternatively, the form of Maxwell's Equations that govern a trapped $\mathrm{EM}$ wave in $\mathrm{TM}_{\mathrm{mnk}}$ mode propagation field can also be ${ }^{21}$

$$
\partial^{2} \mathrm{E}_{\mathrm{z}} / \partial \mathrm{r}^{2}+\mathrm{r}^{-1} \partial \mathrm{E}_{\mathrm{z}} / \partial \mathrm{r}+\mathrm{r}^{-2} \partial^{2} \mathrm{E}_{\mathrm{z}} / \partial \phi^{2}+\mathrm{k}_{\mathrm{c}}^{2} \mathrm{E}_{\mathrm{z}}=0
$$

with eigenvalues

$$
\mathrm{k}_{\mathrm{c}}^{2}=\gamma^{2}+\omega^{2} \epsilon \mu
$$

where one possible solution is

$$
\mathrm{E}_{\mathrm{z}}=\mathrm{E}_{1} \mathrm{~J}_{\mathrm{m}}\left(\mathrm{k}_{\mathrm{c}} \mathrm{r}\right) \cos \mathrm{m} \phi
$$

with $\mathrm{J}_{\mathrm{m}}\left(\mathrm{k}_{\mathrm{c}} \mathrm{r}\right)$ as a Bessel function of the first kind of order $m$ with argument $\mathrm{k}_{\mathrm{c}}$, and $\mathrm{E}_{1}$ as a constant for propagated energy magnitude with $\left[E_{z}\right]_{r=a}=0$ at the walls. The solution in Eq. (38) leads to a field distribution form, $\mathrm{TM}_{01}$ that can then be mapped to a proton as an analog. In such a case, the $\mathrm{TM}_{011}$ mode (see ref 21-Fig. 4-7) cross-section distribution of the magnetic field is circular and contained within the particle whereas the electric field distribution spreads radially and externally from the center. A quark form model is also available as $\mathrm{TM}_{013}$ mode with three similar tiers. Thus, a static proton analog has only observable charge.

A conceivable scenario for Beta Decay in an isolated neutron population is a collision process caused by its magnetic moment property that converts a TE 01 mode neutron resonance to a lower energy $\mathrm{TM}_{01}$ proton resonance mode. Such an EM resonance based state change corresponds to the QCD "color change" concept.

\subsection{Quantum Electrodynamics}

The traditional method for analytically modeling the interaction between a photon and a charged particle is the behavioral propagator based procedure introduced by Feynman in the mid-twentieth century. As shown in the text by Greiner and Reinhardt, such a propagator procedure can be viewed as a linear approximation of an adiabatic particle collision process. ${ }^{19}$ Thus, the Feynman propagator is applicable only to weak interactions. A less complex method that makes use of a microwave analog that yields similar results with less analytical effort, however, was introduced by our group in 2010. ${ }^{23}$ As shown above, the orbital kinetic energy of the electron in the Hydrogen atom the Schrödinger equation in Eq. (12) is 15

$$
\mathrm{KE}_{\text {orbital }}=1 / 2 \mathrm{mv}_{\text {orbital }}{ }^{2}=1 / 2 \mathrm{Lv}_{\text {orbital }}=l(l+1) \hbar^{2} / 2 \mathrm{mr}^{2}
$$

Viewed as a microwave analog, the orbiting electron moves with velocity $\mathrm{v}_{\text {orbital }}$ in a loss-less closed circular traveling wave tube, synchronously as a resonance trapped propagating EM field. Therefore, the number of full wave spins in the traveling EM wave is defined by the spin/orbit coupling constant attribute. Electron orbital velocity therefore is ${ }^{23}$

$$
\mathrm{v}_{\text {orbital }}=[l(l+1)]^{1 / 2} \mathrm{~h} / \mathrm{mr}=\mathrm{c} / \mathrm{N}=\mathrm{có} / \mathrm{n},
$$

where the constant $\mathrm{N}$ restricts the resonance the EM traveling wave to a set of orbital states that are constrained by Planck 
constant $\hbar$. Note also that $\alpha$ is the reduced mass corrected fine structure (spin/orbit coupling) constant for the orbiting electron is defined by

$$
\dot{\alpha}=\alpha[\mathrm{M} /(\mathrm{M}+\mathrm{m})]^{1 / 2}=(137)^{-1},
$$

Photon and charged particle interaction attributes of the orbiting electron can then be defined in terms of total energy 23

$$
\mathrm{E}_{\mathrm{n}}=\mathrm{KE}_{\text {radial }}+\mathrm{KE}_{\text {orbital }}+\mathrm{V}+\mathrm{E}_{\text {self }}=\mathrm{E}_{1} / \mathrm{n}^{2}=\mathrm{má}^{2} \mathrm{c}^{2} / 2 \mathrm{n}^{2}
$$

where $K E_{\text {radial }}=0$ (eg; no radial kinetic motion ), $K E_{\text {orbital }}=l(l+1) m \dot{\alpha}^{2} c^{2} / 2 n^{2}$, the potential energy $V=m \dot{\alpha}^{2} c^{2} / n$, and $m \alpha^{2} \mathrm{c}^{2}, E_{\text {self }}$ the self-energy due to the orbiting electron interacting with its own local EM field. When the electron is in a $p$ state (eg; $l=1)$, Eq. (42) reduces to:

$$
\mathrm{E}_{\mathrm{n}}=0+\mathrm{m} \dot{\alpha}^{2} \mathrm{c}^{2} / \mathrm{n}^{2}-\mathrm{m} \dot{\alpha}^{2} \mathrm{c}^{2} / \mathrm{n}+\mathrm{E}_{\mathrm{self}}=\mathrm{m} \dot{\alpha}^{2} \mathrm{c}^{2} / 2 \mathrm{n}^{2} \text {. }
$$

While in a $p$ state $(\mathrm{eg} ; l=1)$ and the orbiting electron, moving at velocity $\alpha \mathrm{c} / \mathrm{n}^{2}$, self-energy component therefore is

$$
\mathrm{E}_{\mathrm{self}}=\mathrm{m} \dot{\alpha}^{2} \mathrm{c}^{2}\left[(2 \mathrm{n}-3) /\left(2 \mathrm{n}^{2}\right)\right] .
$$

Consequently, when the electron is in a $2 p_{1 / 2}$ state at velocity ác/4 (eg; $n=2)$; self-energy component is at a maximum, and thus, Eq. (44) reduces to:

$$
\mathrm{E}_{\mathrm{self}}=\mathrm{má}^{2} \mathrm{c}^{2} / 8 \text {. }
$$

Such a self energy result is consistent with that from a Feynman Rules based QED analysis procedure. ${ }^{23,24}$

The Lamb Shift" split between the $2 \mathrm{~s}_{1 / 2}$ and $2 \mathrm{p}_{1 / 2}$ energy states of an orbiting electron can also be resolved using an approximation of the Feynman propagator model for the Dirac equation in a Coulomb field. After much analytical effort, the result is predicted to be ${ }^{24}$

$$
\left[\delta \mathrm{E}\left(2 \mathrm{~s}_{1 / 2}\right)-\delta \mathrm{E}\left(2 \mathrm{p}_{1 / 2}\right)\right] / \mathrm{h} \approx+1052.2 \mathrm{Mhz} .
$$

The result in Eq. (46) compares quite well with a relatively recent "Lamb Shift" energy shift observation of +1057.845 Mhz that includes a number of higher order components ${ }^{25,26}$ where $\delta E\left(2 s_{1 / 2}\right) \approx+4.29828 \times 10^{-6} \mathrm{eV}$ or $+1039.3 \mathrm{Mhz}$ and $\delta E\left(2 p_{1 / 2}\right) \approx-5.328 \times 10^{-8} \mathrm{eV}$ or $-12.9 \mathrm{Mhz}$

Now, using a new procedure introduced by our group to determine "Lamb Shaift", ${ }^{23}$ it should be noted that the obvious difference between the $2 \mathrm{~s}_{1 / 2}$ and $2 \mathrm{p}_{12}$ energy states concerns Orbital quantum number. The Orbital quantum number is $l=0$ in the $2 \mathrm{~s}_{12}$ state, and $l=1$ in the $2 \mathrm{p}_{12}$ state. Since both orbital and spin are relevant, analysis begins with the Dirac QM solution: ${ }^{27}$

$$
E_{n j}=E_{n}\left[1+(\alpha / n)^{2}\left(n(j+1 / 2)^{-1}-3 / 4\right)\right],
$$

where $E_{n}=-(m / 2)(\alpha c / n)^{2}$. It can be seen from Eq. (47) then that total energy of the orbiting electron in the $n=2$ state is

$$
\mathrm{E}_{2}=\mathrm{E}_{1} / 4=-(\mathrm{m} / 2)(\alpha \mathrm{c} / 2)^{2} \approx-13.60 / 4 \mathrm{eV} \approx-3.40 \mathrm{eV} .
$$

In the $2 p_{1 / 2}$ state, however, to be consistent with the "reduced mass" $\alpha / \alpha$ correction defined in Eq.(41), total energy of the electron requires a "reduced mass" $\alpha^{2}$ to $\alpha^{2}$ energy shift correction for the $\alpha^{2}$ term in Eq. (47) when $l=1$. Using the identity for small $x$ : ${ }^{23}$

$$
(1-\mathrm{x})^{1 / 4} \approx 1-\mathrm{x} / 4,
$$

the correction to Eq.(47) for the $\alpha^{2}=\alpha^{2}(1836 / 1837)^{1 / 4}=\alpha^{2}[1-$ $(1 / 1837)]^{1 / 4}$ shift is approximately

$$
\Delta \mathrm{E}_{2} \approx \mathrm{E}_{2} /[4(1837)] \text {. }
$$

Then, assuming orbital and spin angular momentum are anti-parallel, in the $2 \mathrm{p}_{1 / 2}$ state with $n=2, l=1$, and $j=1 / 2$, the total electron energy from Eq.(47) becomes ${ }^{23}$

$$
\mathrm{E}\left(2 \mathrm{p}_{1 / 2}\right)=-(\mathrm{m} / 2)(\dot{\alpha} \mathrm{c} / 2)^{2}\left[1+(\dot{\alpha} / 2)^{2}(1.25)\right] \mathrm{eV}
$$

Alternatively, in the $2 \mathrm{~s}_{1 / 2}$ state with $n=2, l=0$, and $j=1 / 2$, total energy of the orbiting electron is

$$
\left.\mathrm{E}\left(2 \mathrm{~s}_{1 / 2}\right)=-(\mathrm{m} / 2)(\alpha \mathrm{c} / 2)^{2}\left[1+(\alpha / 2)^{2} 1.25\right)\right] \mathrm{eV} .
$$

The $2 \mathrm{~s}_{1 / 2}$ and $2 \mathrm{p}_{1 / 2}$ energy split is therefore

$$
\begin{gathered}
\mathrm{E}\left(2 \mathrm{~s}_{1 / 2}\right)-\mathrm{E}\left(2 \mathrm{p}_{1 / 2}\right)=\Delta \mathrm{E}_{2}\left[\left(1+1.25(\alpha / 2)^{2}\right)-\right. \\
\left.\left(1+1.25(\dot{\alpha} / 2)^{2}\right)\right] \approx+4.31 \times 10^{-6} \mathrm{eV},
\end{gathered}
$$

or equivalently, a "Lamb Shift" frequency change of

$$
\left[\mathrm{E}\left(2 \mathrm{~s}_{1 / 2}\right)-\mathrm{E}\left(2 \mathrm{p}_{1 / 2}\right)\right] / \mathrm{h} \approx+1042 \mathrm{Mhz} \text {. }
$$

The frequency shift in Eq.(54) differs from the above cited observed value of $+1057.845 \mathrm{Mhz}$ by $-15 \mathrm{Mhz}$, which is also almost the same as the $\delta \mathrm{E}\left(2 \mathrm{p}_{1 / 2}\right)$ self-energy $-12.9 \mathrm{Mhz}$ shift in Eq. (46). It should be noted, however, the result in Eq.(54) from the Dirac solution in Eq.(47) does not include the selfenergy impact of a slight shift in orbit locus. Therefore, the $\delta \mathrm{E}\left(2 \mathrm{p}_{1 / 2}\right)$ energy shift must be added to the result in Eq.(54). The updated total "Lamb Shift" result then is ${ }^{23}$

$$
\left[\mathrm{E}\left(2 \mathrm{~s}_{1 / 2}\right)-\mathrm{E}\left(2 \mathrm{p}_{21 /}\right)+\delta \mathrm{E}\left(2 \mathrm{p}_{1 / 2}\right)\right] / \mathrm{h} \approx+1054.9 \mathrm{Mhz}
$$

which compares well with the observed value cited above.

\section{Discussion and Conclusions}

The presentation given here describes a causally based electromagnetic resonance method for analyzing quantum theory attributes. An electromagnetic resonance template, derived from Maxwell's Equations that maps theoretical resonance forms to specific particle and photon attributes, is used with constraint conditions to obtain analytical solutions to problems usually solved via traditional QM, QCD, and QED behavioral procedures. The constraints include integer spin-orbit ratio, integer multiples of Planck's constant, angular momentum balance, charge balance, as well as EM analog form. Results obtained with the new approach are found to be substantially the same as those using traditional solution methods.

Solutions from the Schrödinger equation compare well 
with those from an EM resonance template. The analytical results from the template agree with QM Principal, Orbital and Magnetic quantum numbers, but question the stability of the $L=[l(1-1)]^{1 / 2} h$ angular momentum result from the Schrödinger model, because of a fractional value in $h$. Note here, that the template based EM resonance analysis method described is limited to evaluating only stable resonance forms

Solutions from the EM resonance template, using Compound Resonance forms, are successfully derived for QCD theory conjectured quark mass and charge attributes. Results from a prior publication involving half-spin particles as micro-black-holes also support the QCD "colorconfinement" concept, but point to a relativistic causal basis. Whereas the QCD model depends upon a new $\mathrm{W}^{-}$boson with significant mass to mediate "color-change", EM resonance results involve a particle collision event due to the natural magnetic properties of an isolated neutron population, that change a neutron-like EM TE ${ }_{011}$ resonant cavity mode to a proton-like $\mathrm{TM}_{011}$ mode form. Note that, since QCD is colorconfined", observed results obtained from current collision experiments are necessarily limited.

EM resonance based QED results are the same as those from Feynman based rules based analysis, but obtained with the aid of a microwave analog that significantly reduces analytical effort. EM resonance results are given for orbiting electron Self Energy, and the "Lamb Shift" split between the $2 \mathrm{~s}_{1 / 2}$ and $2 \mathrm{p}_{1 / 2}$ energy states of an orbiting electron in a Hydrogen atom.

A unanswered question remains: Does the new EM based template based analytical method describe a causal basis for quantum theory, or is it simply another behavioral model? Analytically, cosmological considerations favor a causal basis. However, current laboratory facilities are not yet sufficient to produce conclusive observed data results that would resolve the issue.

\section{Acknowledgements}

The author wishes to express gratitude for the encouragement and critical review received during the early stages of the work described here from E.J. Bacinich, Director of the Alpha Omega Research Foundation, and several other members of our group, now all deceased: Prof. Behram Kursunoglu and Dr. Walter Rosenthal. Prof. Kursunoglu was the Director of the Theoretical Institute at the University of Miami (FL), and pursued graduate studies in Physics at Cambridge University under P.A.M. Dirac. Dr. Rosenthal was one of the last students to pursue graduate studies in Physics at the University of Berlin under Max Planck.

\section{References}

[1] A. Beiser, Concepts of Modern Physics (fifth edition), (McGraw-Hill, New York 1995), p. 221.
[2] A. Einstein, B. Podolsky, and N. Rosen, Phys. Rev., 47(10), p.777, (1935).

[3] P.J.E. Peebles, Quantum Mechanics, (Princeton University Press, Princeton, NJ 1992), Ch. 4.

[4] M. Planck, "Über eine Verbesserung der Wienschen Spektralgleichung", (Verhandlungen der Deutschen Physikalischen Gesellschaft, Berlin, 1900) 2: pp.202-204.

[5] A. Beiser, Concepts of Modern Physics (fifth edition), (McGraw-Hill, New York 1995), pp. 56-59.

[6] A. Beiser, Concepts of Modern Physics (fifth edition), (McGraw-Hill, New York 1995), pp. 91-93.

[7] L. de Broglie, Recherches sur la théorie des quanta, (Thesis, University of Paris 1924).

[8] E. Schrödinger, Ann der Physik, 79, 361 (1926).

[9] Robert H. Dicke biography, (Encyclopedia Britannica 2014)

[10] T.A. Kriz and E.J. Bacinich, Phys Essays 22, 581, (2009).

[11] T.A. Kriz and E.J. Bacinich, Phys Essays 21, 273, (2008).

[12] A. Beiser, Concepts of Modern Physics (fifth edition), (McGraw-Hill, New York 1995), pp. 202-210.

[13] A. Beiser, Concepts of Modern Physics (fifth edition), (McGraw-Hill, New York 1995), pp. 206-208.

[14] R.P. Feynman, QED - The Strange Theory of Light and Matter, (Princeton University Press, 1985), p.129.

[15] A. Beiser, Concepts of Modern Physics (fifth edition), (McGraw-Hill, New York 1995), pp. 139-140.

[16] M. Gell-Mann, Phys. Lettt. 8, 214 (1964).

[17] G. Zweig, CERN Rpt \#8182/TH.401, (1964).

[18] T.A. Kriz, American J. of Modern Phys., 4(1), 10-14 (2015).

[19] W. Greiner and J. Reinhardt, Quantum Electrodynamics (third edition), (Springer, Berlin 2003), Ch. 2.

[20] M. Dine, Supersymmetry and String Theory, (Cambridge University Press. Cambridge. 2007), Ch. 3.

[21] T.K. Ishii, Microwave Engineering (second edition), (Harcourt Brace Jovanovich, San Diego, CA 1989), S. 4-2.

[22] R.P. Feynman, QED - The Strange Theory of Light and Matter, (Princeton University Press, 1985), p.131.

[23] T.A. Kriz, Phys Essays 25, 221, (2012).

[24] W. Greiner and J. Reinhardt, Quantum Electrodynamics (third edition), (Springer, Berlin 2003), S. 5.3.

[25] W. Greiner and J. Reinhardt, Quantum Electrodynamics (third edition), (Springer, Berlin 2003), S. 5.9.

[26] S.R. Lundeen and F.M. Pipkin, Phys. Rev. Lett., 46, 232 (1981).

[27] R. Eisberg and R. Resnick, Quantum Physics of Atoms, Molecules, Solids, Nuclei, and Particles (second edition), (Wiley, New York 1985), pp.284-286. 